\title{
Japan begins processing data for genome programme
}

Tokyo. An important element in Japan's efforts to process data from the human genome project was last week opened to outside users. The Human Genome Centre of Tokyo University's Institute of Medical Science is one of two central dataprocessing facilities - the other being the Supercomputer Laboratory of Kyoto University - in a rapidly expanding computer network for handling genome data.

The centre, located in a new, two-storey building at the back of the Institute of Medical Science, is not as grand as what was described in a 1990 plan by a committee of nearly 70 scientists headed by Kenichi Matsubara of Osaka University. Its recommendation for eight laboratories and a permanent staff of 36 was reduced to three laboratories by the Ministry of Education, Science and Culture, which runs Japan's national universities (see Nature 349; 360; 1991).

Two are operating: the Laboratory of Genome Database, which last week opened its computer system to outside users, and the Laboratory of Genome Structure Analysis. The third, the Laboratory of DNA Information Analysis, will begin work in April. About 15 scientists, including students, are in the building, with some members of the genome structure analysis laboratory working in temporary quarters elsewhere. Plans for a supercomputer were dropped after a 1990 US-Japan trade agreement severely limited the number of supercomputers the ministry can buy each year and the centre decided not to wait in the queue (see below).

Nevertheless, there is an impressive array of hardware on hand, most of it from the United States and leased at an annual cost of $¥ 300$ million ( $\$ 2.4$ million). There is a nCUBE2 parallel computer with 64 processors and a CM-5 parallel computer with 32 processors to develop parallel algorithms for handling genome data, powerful database servers (SUN SPARCstation 690MP) and computation servers (IRIS Crimson VGXT), as well as numerous graphics and engineering workstations. The only large piece of hardware from Japan is the Hitachi HITAC M-860/160 file server.

The genome centre is joined by a highcapacity ( 1.5 megabit per second) computer link to the faculty of science on the main campus of Tokyo University on the other side of the city. From there, the centre's computers can tap into the rapidly expanding Todai International Science Network (see Nature 356, 550; 1992), which provides access to the US network Internet and US databases, such as the Genome Data Base (GDB) and GenBank. The centre's scientists have developed their own integrated database system called HyperGenome to retrieve information on genetic maps stored in GDB and sequence data stored in GenBank; this database is available free to outside users.

The centre's wide-area network, called "GenomeNet", has high-capacity links to five other centres in Japan. GenomeNet is also linked to another high-capacity network, the Widely Integrated Distributed Environment (WIDE), which is used mainly by researchers developing computer networks but also provides university scientists with access to overseas networks.

The supercomputer laboratory at the Institute for Chemical Research (ICR) in Kyoto, the other main centre of GenomeNet, complements the Tokyo centre; Kyoto offers powerful computation capability and Tokyo will develop advanced databases and algorithms with its parallel computers. Minoru Kanehisa, head of the genome database laboratory and a professor at ICR,

\section{Trade agreement backfires}

Tokyo. The 1990 trade agreement between the United States and Japan, rather than increasing sales of US supercomputers to Japan's universities and national institutes as intended, has made it harder for Japanese institutions to buy supercomputers from any source.

Before the agreement, universities were buying about five supercomputers a year at a large discount from Japanese companies. But the agreement eliminated the discounts, and the Ministry of Education, Science and Culture now approves the purchase of only two or three supercomputers a year. As a result, universities are having to wait several years, or, as in the case of the Human Genome Centre, buy less powerful machines.

Universities also hesitate to buy supercomputers for fear of offending a competitor. For example, when the National institute of Fusion Science selected a NEC supercomputer, Cray Research of the United States filed a formal complaint based on allegations that NEC exaggerated the machine's performance (see Nature 358, 265; 1992).

Since the agreement, Cray has sold only three supercomputers to Japan's public sector, while its sales to Japan's private sector over the same period are much larger. D.S.

Researchers like US products

Tokyo. A striking feature of the new Human Genome Centre is how much of its equipment is not Japanese. Not only are most of the computers US-made (see left), but also a pilot semiautomatic sequencing system in the Laboratory of Genome Structure Analysis consists largely of US and European machines.

Japan's Science and Technology Agency has invested several million dollars over the past ten years in automatic DNA sequencing machines in collaboration with Japanese companies such as Seiko and Hitachi (see Nature 351, 593; 1991). But given a choice, it seems that Japan's genome researchers prefer machines made by Applied Biosystems Inc. of California and Pharmacia of Sweden.

The pilot system at the centre has a much smaller capacity than the system run by Craig Venter, who last summer left the US National Institutes of Health (NIH) to run a new, private sequencing company. But the laboratory hopes to build a much larger system if the centre gets the money to move to a larger building.

D.S.

divides his time between the two facilities.

Kanehisa expects the number of Japanese users eventually to reach a thousand, most of them at universities and national laboratories. But fewer than two dozen tapped into the facility on opening day.

A lack of money has already delayed one important component of the network. In 1991, the Science and Technology Agency, a rival of the education ministry, applied for funds for the fiscal year beginning 1 April 1992 to establish a high-capacity international computer link between the Japan Information Centre of Science and Technology (JICST) in Tokyo and GDB at Johns Hopkins University in the United States. Under the plan, JICST would be linked to the genome centre.

But the Ministry of Finance rejected the request for administrative reasons, and later awarded to the agency's Institute of Physical and Chemical Research only $¥ 112$ million of the initial request of $¥ 280$ million. One consequence is that the international link to GDB cannot begin before January 1994.

Sanzo Miyazawa of Gumma University, who is helping to establish the JICST network, says that JICST will be connected to the genome centre and other networks and will serve private companies and national laboratories, while the genome centre will be used mainly by university researchers. But how that will work in practice remains unclear.

David Swinbanks 